\title{
Identification of cisplatin sensitizers through high-throughput combinatorial screening
}

\author{
YICHEN SUN $^{1 *}$, WEIYE JIANG $^{1 *}$, WENHUA LU $^{1}$, MING SONG $^{1}$, KAIYAN LIU $^{1}$, PING CHEN $^{1}$, \\ ALLISON CHANG ${ }^{2}$, JIANHUA LING ${ }^{2}$, PAUL J. CHIAO ${ }^{2}$, YUMIN HU ${ }^{1}$ and PENG HUANG ${ }^{1,3}$ \\ ${ }^{1}$ Sun Yat-sen University Cancer Center, State Key Laboratory of Oncology in South China, \\ Collaborative Innovation Center for Cancer Medicine, Guangzhou, Guangdong 510060, P.R. China; \\ Departments of ${ }^{2}$ Molecular and Cellular Oncology, and ${ }^{3}$ Translational Molecular Pathology, \\ The University of Texas MD Anderson Cancer Center, Houston, TX 77030, USA
}

Received January 11, 2018; Accepted May 15, 2018

DOI: 10.3892/ijo.2018.4447

\begin{abstract}
Diamminedichloroplatinum/cisplatin (CDDP) is a major drug used in cancer chemotherapy; however, the toxic side-effects and development of drug resistance represent major challenges to the clinical use of CDDP. The aim of the present study was to identify effective drug combination regimens through high-throughput drug screening that can enhance the efficacy of CDDP, and to investigate the underlying mechanisms. A cell-based high-throughput screening methodology was implemented, using a library of 1,280 Food and Drug Administration (FDA)-approved drugs, to identify clinical compounds that act synergistically with CDDP. Our study identified two compounds, namely potassium antimony tartrate and topotecan, that significantly enhanced the sensitivity of colorectal and non-small cell lung cancer cells to CDDP. The synergistic action of both compounds with CDDP was confirmed by further quantitative analyses. Topotecan is a topoisomerase-1 inhibitor that has previously been shown to enhance the clinical response and overall patient survival when combined with CDDP by a yet unclear mechanism. We demonstrated that the combination of topotecan with CDDP significantly inhibited colony formation ability and increased the apoptosis of several cancer cell lines. Mechanistic analyses revealed that topotecan enhanced CDDP-induced DNA
\end{abstract}

Correspondence to: Professor Peng Huang or Dr Yumin Hu, Sun Yat-sen University Cancer Center, State Key Laboratory of Oncology in South China, Collaborative Innovation Center for Cancer Medicine, 651 Dongfeng Road East, Guangzhou, Guangdong 510060, P.R. China

E-mail: huangpeng@sysucc.org.cn

E-mail: huym@sysucc.org.cn

${ }^{*}$ Contributed equally

Abbreviations: CDDP, cis-diamminedichloroplatinum/cisplatin; PAT, potassium antimony tartrate; HTS, high-throughput screening

Key words: cisplatin, high-throughput screening, PAT, topotecan, drug combination, DNA damage damage and inhibited the repair of DNA strand breaks, without affecting the cellular platinum content. Overall, the findings of this study demonstrated that the use of the FDA-approved drug panel in high-throughput screening is an effective method for identifying effective therapeutic regimens that are clinically relevant, and may have high feasibility for translation into clinical practice.

\section{Introduction}

Cisplatin (cis-diamminedichloroplatinum, CDDP) is a frontline drug used in the clinical treatment of cancers of various tissue types, including colorectal, lung, ovarian, testicular, penile, cervical, head and neck, and bladder carcinomas. CDDP was the first Food and Drug Administration (FDA)-approved platinum (Pt)-based drug for cancer treatment in the 1970s, and continues to be one of the most widely used chemotherapeutic drugs (1-4). CDDP is considered a cytotoxic agent, as it binds and damages DNA, thus triggering various cytotoxic events, such as the inhibition of DNA replication, transcription arrest, DNA damage responses, cell cycle arrest, and apoptosis (5). Such toxic effects limit the clinical application of CDDP-based chemotherapy, which is associated with nephrotoxicity, neurotoxicity and other significant side-effects (6-8). CDDP-induced side-effects are dose-dependent, limiting the administration of the effective dose, thus compromising the therapeutic efficacy (9). The development of drug resistance is another major challenge. Several factors may be responsible for resistance to CDDP, such as reduced cellular uptake, increased efflux and increased DNA repair $(10,11)$. Combining drugs is a potentially effective method for overcoming resistance and minimizing toxic side-effects, as it allows for a lower drug dose. Therefore, effective regimens that can potentiate the therapeutic efficacy of CDDP are urgently required.

Pt-based drugs, including CDDP, carboplatin and oxaliplatin, have been clinically evaluated in combination with other chemotherapeutic agents, including etoposide, mitomycin $\mathrm{C}$, vinblastine, paclitaxel, docetaxel, vinorelbine, gemcitabine, cyclophosphamide, doxorubicin, epirubicin, methotrexate, lonidamine and 5-fluorouracil (12). Several Pt-based drug combination regimens have achieved an improvement in 
clinical response and have positively affected the overall survival of patients $(13,14)$. These drug combinations are largely the results of empirical clinical trials rather than mechanism-based design, as mechanistic studies and clinical evaluation require vast resources (15). In recent years, the evaluation of FDA-approved drugs for new disease indications (drug repurposing) has emerged as an effective strategy for discovering novel therapeutic measures that can be rapidly translated into clinical applications due to the availability of prior knowledge regarding the drug mechanisms of action, pharmacokinetics, formulation, and toxicity/safety information (16-18).

In the present study, we used a cell-based high-throughput screening (HTS) approach to screen the FDA-approved drugs $(1,280$ compounds in total) in search of drugs that potentially act synergistically with CDDP. We identified two compounds, namely potassium antimony tartrate (PAT), and topotecan, that were capable of significantly enhancing the anticancer activity of CDDP. Topotecan was further investigated to gain mechanistic insight into the synergic activity.

\section{Materials and methods}

Chemicals and reagents. The FDA-approved drug library of 1,280 compounds (10 mM concentration in DMSO, 80 compounds/96-well plate, 16 plates) was purchased from MicroSource Discovery Systems (Gaylordsville, CT, USA). CDDP and topotecan were purchased from Hospira (Lake Forest, IL, USA) and GlaxoSmithKline (London, UK), respectively. MTS, PAT and ethanol were purchased from Sigma-Aldrich (Merck KGaA, Darmstadt, Germany).

Cells and cell culture. All the cell lines used in this study were purchased from the American Type Culture Collection (ATCC, Manassas, VA, USA). The human colorectal cancer cell line, DLD-1, and the non-small-cell lung cancer (NSCLC) cell line, NCI-H460, were cultured in RPMI-1640 (Corning Cellgro, Shanghai, China) supplemented with $10 \%$ fetal bovine serum (Invitrogen; Thermo Fisher Scientific, Inc., Waltham, MA, USA). The colon cancer cell lines, HT-29 and HCT-116, were maintained in McCoy's 5A (Sigma-Aldrich) with $10 \%$ fetal bovine serum (FBS). The LS-174T and RKO human colorectal cancer cell lines were cultured in DMEM and MEM (Corning Cellgro) supplemented with $10 \%$ FBS, respectively. The cells were cultured at $37^{\circ} \mathrm{C}$ in a humidified incubator (Thermo Fisher Scientific, Inc.) containing 5\% $\mathrm{CO}_{2}$, and were seeded and incubated in culture flasks or plates overnight prior to each treatment.

Cell viability assay and high-throughput drug screening. Cell viability was measured using the MTS colorimetric assay. Briefly, the cells were seeded in a 96-well plate at a density of 2,000 cells per well, and treated with the indicated drugs at the specified concentrations. Following a 72-h incubation, $20 \mu 1$ of MTS were added to each well and incubated for a further $3 \mathrm{~h}$. The absorbance of each well at $490 \mathrm{~nm}$ was measured using a microplate reader (BioTek Instruments, Inc., Winooski, VT, USA) and each experiment was performed in triplicate wells. The 1,280 FDA-approved drugs were stored as $10-\mathrm{mM}$ solutions in $100 \%$ DMSO and diluted to $2 \mathrm{mM}$ with phosphate-buffered saline (PBS) using a single-arm, multichannel workstation (Aurora Biomed, Inc., Vancouver, BC, Canada). Using this workstation, the high-throughput MTS viability assays were performed as described in Table I.

Numerical characterization method of synergy, additivity and antagonism. The drug combination index (CI) was calculated using the CalcuSyn software developed by Chou (19) (CI $<1$, synergism; $\mathrm{CI}=1$, additive effect; $\mathrm{CI}>1$, antagonism). The methods by which the normalized cell survival rate was characterized were based on a previous publication (20). Given the viability of drugs $A$ and $B$ at the respective concentrations $x$ and $y$ as $V_{A}$ and $V_{B}$, the additive response viability is predicted as $\mathrm{V}_{\mathrm{A}} \mathrm{V}_{\mathrm{B}}$.

Colony formation assay. A total of 500 cells/well were plated in 6-well plates and cultured with each drug for 10-14 days. Each of the colonies was washed twice with PBS, fixed with methanol and stained with crystal violet for $15 \mathrm{~min}$ at room temperature. After washing with water, the colonies were photographed and counted using the AlphaImager HP system (ProteinSimple, San Jose, CA, USA).

Cellular apoptosis assay. The cells were seeded in a 6-well plate as $2 \times 10^{5}$ cells/well, allowed to adhere overnight, and were then treated with the indicated drugs as described in the figure legends. The cells were harvested by trypsinization, washed with PBS $\left(4^{\circ} \mathrm{C}\right)$, and suspended in $500 \mu \mathrm{l}$ buffer. The cells were stained with Annexin V-FITC for $15 \mathrm{~min}$ and then stained with PI (Apoptosis kit from KeyGen Biotech, Nanjing, China) for $5 \mathrm{~min}$ at room temperature. The samples were analyzed by a FACSCalibur flow cytometer (BD Biosciences, Franklin Lakes, NJ, USA).

Comet assay (single-cell gel electrophoresis assay). A Comet assay was performed according to the method previously described (21). Briefly, following treatment with the indicated drugs for $6 \mathrm{~h}$, the cells were collected, washed and re-suspended in cold PBS. The cell suspensions $\left(2 \times 10^{6} \mathrm{cell} / \mathrm{s} / \mathrm{ml}\right.$, $20 \mu 1$ ) were mixed with $100 \mu 10.5 \%$ low-melting-point agar (Sigma-Aldrich), and were then placed onto a slide pre-coated with $1 \%$ normal-melting-point agar (Sigma-Aldrich). When the agar was solidified, the slides were submerged in fresh prechilled lysis buffer (10 mM Tris- $\mathrm{HCl}, \mathrm{pH} 10.0,2.5 \mathrm{M} \mathrm{NaCl}$, $100 \mathrm{mM}$ ethylenediaminetetraacetic acid and $1 \%$ Triton X-100) for at least $1 \mathrm{~h}$ at $4^{\circ} \mathrm{C}$. After rinsing with a neutralization buffer (0.4 M Tris- $\mathrm{HCl}, \mathrm{pH} 7.5)$ for $5 \mathrm{~min}$, the slides were soaked in the alkaline electrophoresis buffer $(300 \mathrm{mM} \mathrm{NaOH}, 1 \mathrm{mM}$ ethylenediaminetetraacetic acid, $\mathrm{pH}>13,4^{\circ} \mathrm{C}$ ) for $15 \mathrm{~min}$ and then subjected to electrophoresis for a further $15-20 \min (25 \mathrm{~V}$, $300 \mathrm{~mA}$ ). The slides were then stained with SYBR-Green I (Biotek, Beijing, China) and photographed under a fluorescence microscope (Nikon, Tokyo, Japan). The CASP software program, version 1.2.2, provided by the CASPLab Comet Assay Project, was used to analyze the percentage of tail DNA, which indicates damaged DNA.

Cellular Pt content analysis. The measurement of the total intracellular Pt content was performed as previously described (22). In brief, following CDDP treatment, the cells 
A

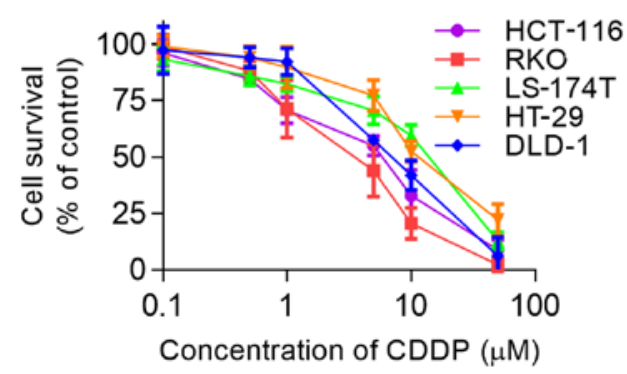

C

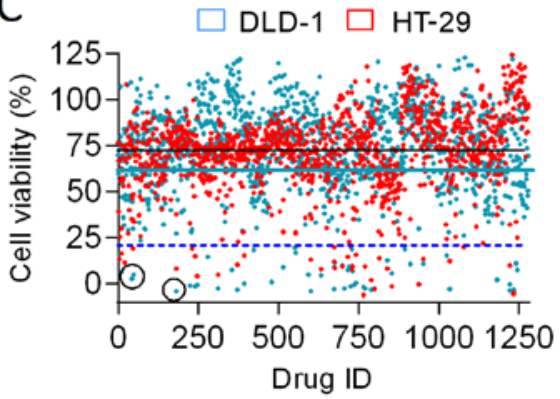

B

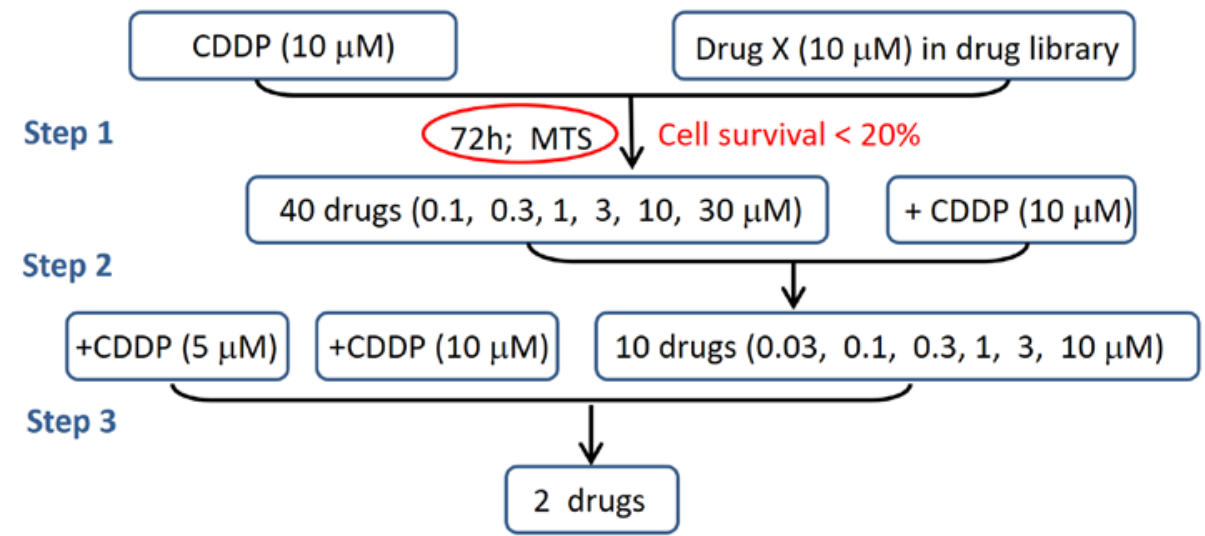

Figure 1. Cell-based high throughput screening (HTS) of FDA-approved drug library for sensitizers of cisplatin. (A) MTS assay of the sensitivity of 5 colorectal cancer cell lines to cisplatin (CDDP). Data are shown as the means $\pm S D, n=3$ experiments. (B) A schematic illustration of the combination screening for drugs having synergistic effect with CDDP. Each of the compounds (drug X, $10 \mu \mathrm{M})$ in the FDA-approved drug library (1,280 compounds) was tested in the first round screening (Step 1) in combination with $10 \mu \mathrm{M}$ CDDP. The preliminary hits ( 40 compounds) were further tested in Steps 2 and 3 for synergistic effects in combination with CDDP, using multiple drug concentrations. (C) HTS data of 1,280 drugs combined with CDDP using DLD-1 and HT-29 cells. The blue and black lines indicate the survival rates of DLD-1 and HT-29 cells, respectively, treated with $10 \mu \mathrm{M} \mathrm{CDDP}$; the blue dashed line indicate the threshold $(<20 \%$ viable cells) for the primary screening in Step 1 . The dots under the dashed line show potential hits; the two circles indicate the two compounds (PAT and topotecan) validated in Step 3.

were collected, washed twice with cold PBS and counted. Subsequently, $1 \mathrm{ml}$ nitric acid was added to the cell pellets, mixed and kept at $68^{\circ} \mathrm{C}$ for $1 \mathrm{~h}$. The solution was diluted to $1: 10$ with $\mathrm{ddH}_{2} \mathrm{O}$ and sent for a $\mathrm{Pt}$ content analysis using an Agilent 7500ce inductively coupled plasma-mass spectrometer (ICP-MS; Agilent Technologies, Inc., Santa Clara, CA, USA).

Western blot analysis and antibodies. The cells were washed twice with cold PBS and lysed in radio immunoprecipitation assay (RIPA) lysis buffer containing protease inhibitors and phosphatase inhibitors (Cell Signaling Technology, Inc., Danvers, MA, USA) on ice for $20 \mathrm{~min}$. Protein $(30 \mu \mathrm{g})$ was separated by electrophoresis using $10 \%$ SDS-PAGE and transferred onto polyvinylidene fluoride membranes (Millipore, Billerica, MA, USA). The membranes were incubated with mouse anti- $\gamma \mathrm{H} 2 \mathrm{AX}(\mathrm{ab} 22551$, dilution, $1: 2,000)$ at $4^{\circ} \mathrm{C}$ overnight on a shaking platform, washed, and then incubated with horseradish peroxide-conjugated goat anti-mouse secondary antibody (ab6789; dilution, 1:10,000) for $1 \mathrm{~h}$ at room temperature. The blots were visualized using chemiluminescent reagents (KeyGen Biotech) and revealed with X-ray film. $\alpha$-tubulin was used as a loading control. The $\alpha$-tubulin antibody (GTX628802; dilution, 1:5,000) was purchased from GeneTex (Irvine, CA, USA), and all other antibodies were purchased from Abcam (Cambridge, MA, USA).
Statistical analysis. The cytotoxic combined effect of PAT/topotecan and CDDP was calculated using CalcuSyn software (Biosoft, Cambridge, UK). The data were analyzed with GraphPad Prism 5 (GraphPad Software, Inc., La Jolla, CA, USA). A Student's t-test was used to analyze the statistical difference between two groups, while a two-way ANOVA with Bonferroni's correction were used to determine the statistical difference among multiple groups, as indicated in the figure legends. The data are reported as the means \pm SD of 3 independent experiments. The results were deemed statistically significant at a value of $\mathrm{P}<0.05$.

\section{Results}

Screening of drugs that potentiate the anticancer activity of $C D D P$. To identify drugs that may enhance the effects of CDDP, a quantitative cell-based drug screening approach was used to assess the combinational activity of CDDP and one of the 1,280 FDA-approved drugs. The sensitivity to CDDP was first evaluated in several colorectal cancer cell lines to select the optimal drug concentrations and cell lines for the screening. Two cell lines with relatively low sensitivities to CDDP, HT-29 and DLD-1, were selected. As shown in Fig. 1A, the $\mathrm{IC}_{50}$ value for CDDP was $\sim 10 \mu \mathrm{M}$ for both cell lines, which was selected for further combination analyses. Various 
Table I. Drug combination screening protocol used in this study.

\begin{tabular}{|c|c|c|c|}
\hline Step & Parameter & Description & Instrument \\
\hline 1 Drug dilution & $\begin{array}{l}14 \mu 1 \text { Drug X } \\
266 \mu 1 \text { DMSO }\end{array}$ & from $10 \mathrm{mM}$ to $2 \mathrm{mM}$ & $\begin{array}{l}\text { ABV1000 } \\
\text { ABV1000 }\end{array}$ \\
\hline 2 Cell seeding & $100 \mu 1$ & \multicolumn{2}{|l|}{2000 cells/well } \\
\hline 3 Add reagent & $\begin{array}{l}20 \mu 1 \text { Drug } \mathrm{X} \\
80 \mu 1 \mathrm{CDDP}\end{array}$ & $\begin{array}{l}\text { Add two drugs/well } \\
\text { (final concentration: } 10 \mu \mathrm{M} \text { ) }\end{array}$ & ABV1000 \\
\hline 4 Incubate & $72 \mathrm{~h}$ & $5 \% \mathrm{CO}_{2} / 37^{\circ} \mathrm{C}$ incubation & Incubator \\
\hline 5 Add reagents & $20 \mu 1$ & MTS solution $(5 \mathrm{mg} / \mathrm{ml})$ & ABV1000 \\
\hline 6 Incubate & $3 \mathrm{~h}$ & $5 \% \mathrm{CO}_{2} / 37^{\circ} \mathrm{C}$ incubation & Incubator \\
\hline 7 Read & Fluorescence & $490 \mathrm{~nm}$ & Synergy MR \\
\hline \multicolumn{4}{|c|}{$\begin{array}{l}\text { ABV1000, Aurora Biomed Versa } 1000 \text { workstation; Incubator, Forma Series II Water Jacket } \mathrm{CO}_{2} \text { incubator from Thermo Scientific; Synergy } \\
\text { MR, Synergy HT Microplate Reader from BioTek Instruments. }\end{array}$} \\
\hline Rank & Drug name & \multicolumn{2}{|c|}{ Classification } \\
\hline 1 & Antimony potassium tartrate trihydrate & \multicolumn{2}{|c|}{ Antischistosomal } \\
\hline 2 & Topotecan hydrochloride & Antineopla & ase I inhibitor \\
\hline 3 & Thioridazine hydrochloride & \multicolumn{2}{|c|}{ Antipsychotic } \\
\hline 4 & Oxyphenbutazone & \multicolumn{2}{|c|}{ Anti-inflammatory } \\
\hline 5 & Emetine dihydrochloride & \multicolumn{2}{|c|}{ Inhibits RNA, DNA and protein synthesis } \\
\hline 6 & Oxyquinoline sulfate & \multicolumn{2}{|c|}{ Anti-infective, complexing agent } \\
\hline 7 & Monensin sodium (monensin A) & \multicolumn{2}{|c|}{ Antibacterial } \\
\hline 8 & Piroctone olamine & \multicolumn{2}{|c|}{ Antiseborrheic } \\
\hline 9 & Amsacrinea & \multicolumn{2}{|c|}{ Antineoplastic, immune suppressive } \\
\hline 10 & Phenylmercuric acetate & \multicolumn{2}{|c|}{ Antifungal, antimicrobial } \\
\hline
\end{tabular}

experimental conditions, including the number of cells per well, the length of the drug incubation period, and the volume of the reagents, were tested for optimization of the assay. The protocol for the screening study is summarized in Table I. The screening procedures included three main steps, as shown in Fig. 1B. During the primary screening, we compared the viability of the two cell lines exposed to CDDP $(10 \mu \mathrm{M})$ alone or to CDDP in combination with $10 \mu \mathrm{M}$ drug $\mathrm{X}$ (one of the 1,280 drugs in the FDA-approved drug library). A drug that enhanced the CDDP inhibition of cell growth to $<20 \%$ viable cells was considered as a potential hit in the primary screening (Fig. 1C). Using this criterion, 40 drugs capable of enhancing the cytotoxicity of CDDP were identified. Subsequently, the 40 compounds identified from the primary screening were further evaluated at a dose-range of $0.1-30 \mu \mathrm{M}$ to further evaluate the potential synergistic effects in combination with CDDP. The top 10 compounds that were validated in the second test step are listed in Table II. In the third step, the dose-effect analysis described by Chou and Talalay (19) was used to determine the drug CI values of the top hits for the two colon cancer cell lines. Through these 3 steps, we identified two drugs, namely PAT and topotecan, that consistently showed synergy with CDDP.
Synergistic effects of CDDP and topotecan. Since CDDP is often used in the treatment of colon cancer and $\operatorname{NSCLC}(23,24)$, we selected NSCLC (NCI-H460) and colon cancer (DLD-1) cell lines to further investigate the chemosensitization effect of topotecan on CDDP. MTS cell growth inhibition, colony formation and apoptosis assays were used to evaluate the drug combination effects. The results of MTS assay demonstrated that the combination of CDDP and topotecan resulted in a concentration-dependent increase in cell growth inhibition. To examine whether the drug combination was more than additive, cell growth inhibition curves were normalized to the growth inhibition induced by the corresponding concentration (5-10 $\mu \mathrm{M})$ of CDDP alone, according to a previously described method (20). The normalized curves exhibited a significant low-left shift with the drug combination in DLD-1 (Fig. 2A) and NCI-H460 cells (Fig. 2B) respectively, indicating more than an additive effect. The colony formation assay also confirmed a significant loss of more cell colonies when CDDP and topotecan were used in combination in DLD-1 cells (Fig. 2C) and NCI-H460 cells (Fig. 2D). In addition, an apoptosis assay was performed to evaluate the synergistic killing effect of CDDP and topotecan after the DLD-1 cells or NCI-H460 cells were double-stained with 

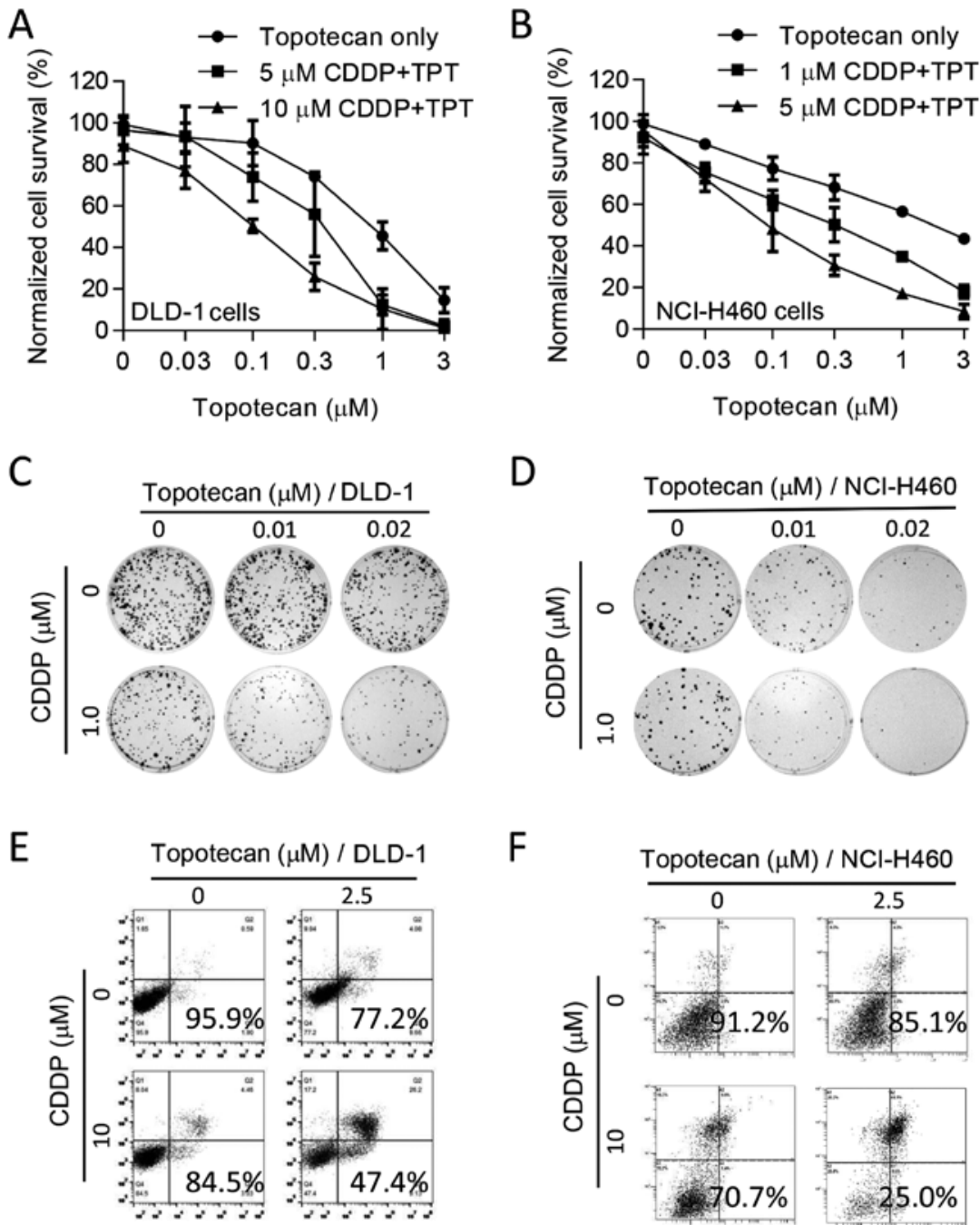

$\mathrm{F}$

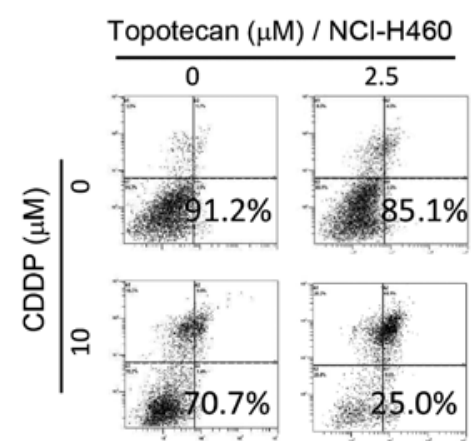

G

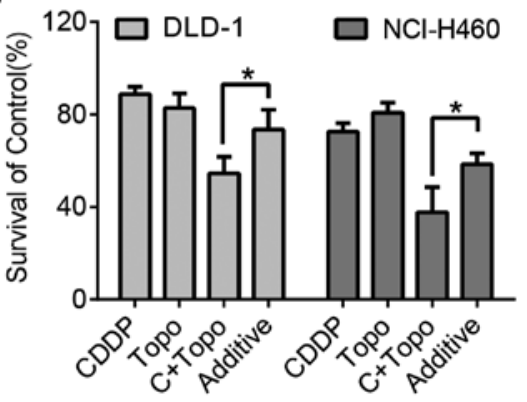

$\mathrm{H}$

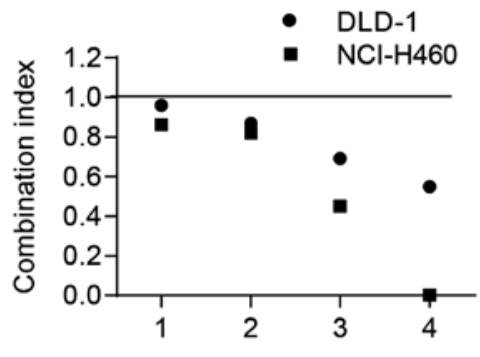

Figure 2. Synergistic effect of cisplatin (CDDP) in combination with topotecan in colon and lung cancer cells. (A) Normalized cell survival curves in DLD-1 cells treated with the the indicated concentrations of topotecan and $\mathrm{CDDP}$ (square, $5 \mu \mathrm{M}$; triangle, $10 \mu \mathrm{M}$ ). Graph shows means $\pm \mathrm{SD}$, $\mathrm{n}=3$ experiments; the $\mathrm{P}$-values for $5 \mu \mathrm{M} \mathrm{CDDP}+$ TPT and $10 \mu \mathrm{M} \mathrm{CDDP}+\mathrm{TPT}$ compared to TPT alone are $2.45 \times 10^{-4}$ and $1.06 \times 10^{-11}$, respectively (two-way ANOVA). (B) Normalized viability of NCI-H460 cells treated with the respective concentration of CDDP alone or combined with topotecan. Graph shows the means \pm SD, $n=3$ experiments; the P-values for $1 \mu \mathrm{M} \mathrm{CDDP}+\mathrm{TPT}$ and $5 \mu \mathrm{M} \mathrm{CDDP}+\mathrm{TPT}$ compared to TPT alone are $1.33 \times 10^{-10}$ and $6.77 \times 10^{-14}$, respectively (two-way ANOVA). (C and D) The colony formation assay of (C) DLD-1 and (D) NCI-H460 cells treated with the indicated concentrations of CDDP and topotecan for 2 weeks. (E and F) Apoptosis induced by CDDP $(10 \mu \mathrm{M})$ and combination treatment with topotecan $(2.5 \mu \mathrm{M})$ for $48 \mathrm{~h}$ was detected by Annexin V/PI double staining followed by flow cytometric analysis in (E) DLD-1 and (F) NCI-H460 cells. (G) Quantitative data of the apoptosis assay in DLD-1 cells and NCI-H460 cells. Additive, indicates the estimated additive effect based on the effect of each drug alone. Data are shown as the means $\pm \mathrm{SD}$. $\mathrm{n}=3$ experiments, "P<0.05. (H) Drug combination index $(\mathrm{CI})$ between CDDP and topotecan in DLD-1 and NCI-H460 cells based on colony formation assay. (CI $<1$, synergism; CI =1, additive; CI $>1$, antagonism).

Annexin V/PI (Fig. 2E and F). Quantitative analysis of triplicate experiments demonstrated that the observed cell survival rate of $54.5 \%$ was significantly lower than the calculated additive effect (73.6\%; P<0.05, Fig. $2 \mathrm{G})$ in the DLD1 cells. A similar synergistic drug combination effect was also observed in the NCI-H460 cells (Fig. 2F and G). Quantitative analysis of the drug combination index revealed that the $\mathrm{CI}$ values were predominately $<1.0$ (Fig. $2 \mathrm{H}$ ), confirming the synergy between CDDP and topotecan.

Enhancement of the CDDP anticancer activity by PAT. Similar to the synergistic effects between CDDP and 

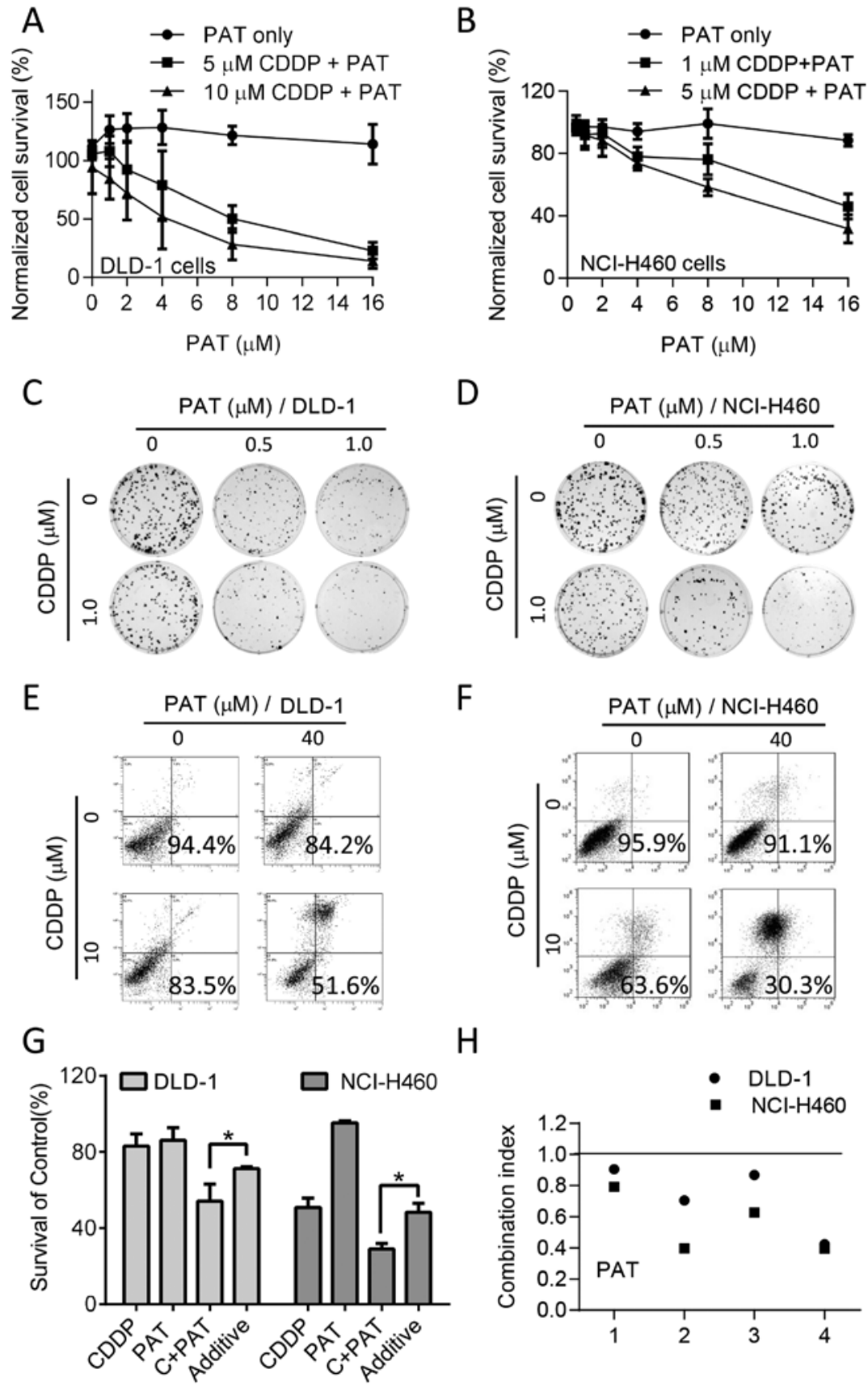

$\mathrm{H}$

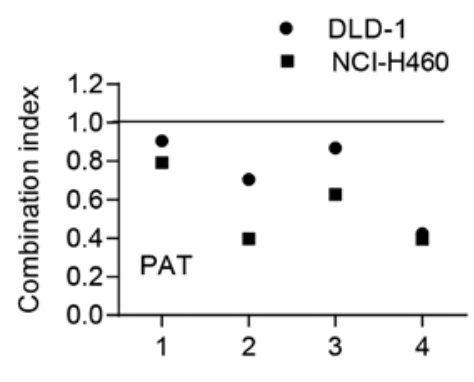

Figure 3. Effect of cisplatin (CDDP) and potassium antimony tartrate (PAT) alone or in combination on the viability of colon and lung cancer cell lines (A) Normalized cell survival curves of DLD-1 colon cancer cells treated with the indicated concentrations of CDDP and PAT. Data are shown as the means $\pm \mathrm{SD}, \mathrm{n}=3$ experiments; The P-values for $5 \mu \mathrm{M}$ CDDP + PAT and $10 \mu \mathrm{M}$ CDDP + PAT compared to PAT alone group are $3.21 \times 10^{-9}$ and $1.67 \times 10^{-11}$, respectively (two-way ANOVA). (B) Normalized cell survival curves of NCI-H460 lung cancer cells treated with the indicated concentrations of CDDP and PAT. Data are shown as the means $\pm \mathrm{SD}, \mathrm{n}=3$ experiments; The P-values for $5 \mu \mathrm{M}$ CDDP + PAT and $10 \mu \mathrm{M}$ CDDP + PAT compared to PAT alone group are $3.82 \times 10^{-4}$ and $3.68 \times 10^{-6}$, respectively (two-way ANOVA). (C and D) The colony formation assay of (C) DLD-1 and (D) NCI-H460 cells treated with the indicated concentration of CDDP, PAT or their combination. (E and F) Induction of apoptosis of (E) DLD-1 and (F) NCI-H460 cells by CDDP, PAT, or their combination for $48 \mathrm{~h}$. (G) Quantitative data of the apoptosis assay in DLD-1 cells. Additive, indicates the estimated additive effect based on the effect of each drug alone. Columns, mean ( $\mathrm{n}=3$ experiments); bars, $\mathrm{SD}$; ${ }^{*} \mathrm{P}<0.05$. (H) Drug combination index (CI) of CDDP and PAT in DLD-1 and NCI-H460 cells (CI $<1$, synergism; $\mathrm{CI}=1$, additive; $\mathrm{CI}>1$, antagonism).

topotecan described above, the ability of PAT to enhance the anticancer activity of CDDP was validated using multiple assays. As is shown in Fig. 3A and B, PAT alone did not exert a significant effect on cell survival within the range of concentrations tested; however, the combination of PAT and CDDP led to a significant decrease in the survival of both the DLD-1 and NCI-H460 cells. The evident low-left shift of the normalized cell survival curves with the drug combination indicated more than an additive effect. Colony formation assay in the DLD-1 (Fig. 3C) and NCI-H460 cells (Fig. 3D) and flow cytometric apoptosis assay (Fig. 3E and F) also confirmed the synergy between CDDP and PAT in both cell lines. All CI values calculated from the colony formation assay with different concentrations of the drug combinations were $<1.0$ (Fig. $3 \mathrm{H}$ ), indicating a synergistic effect of the two drugs in both cell lines. 
A

DLD-1 cells

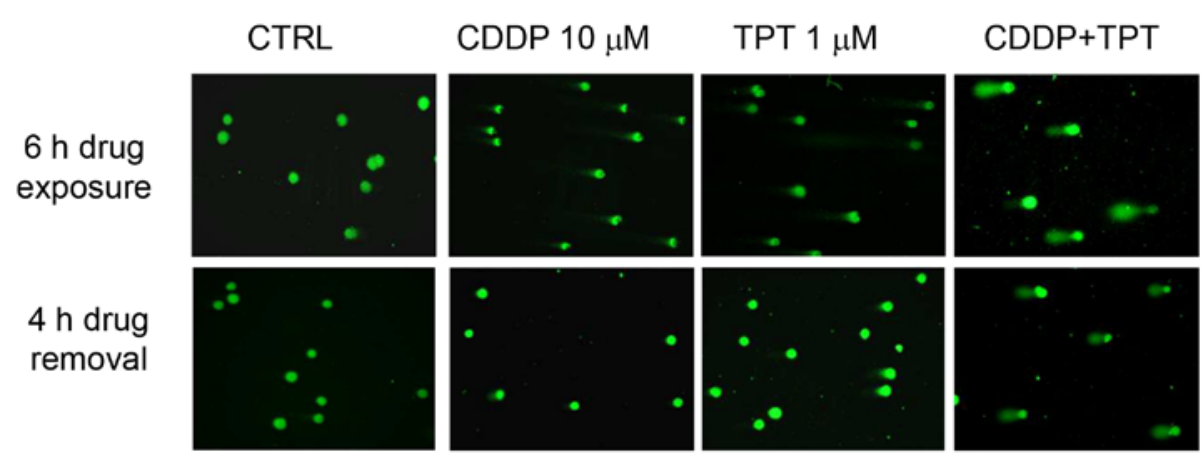

B

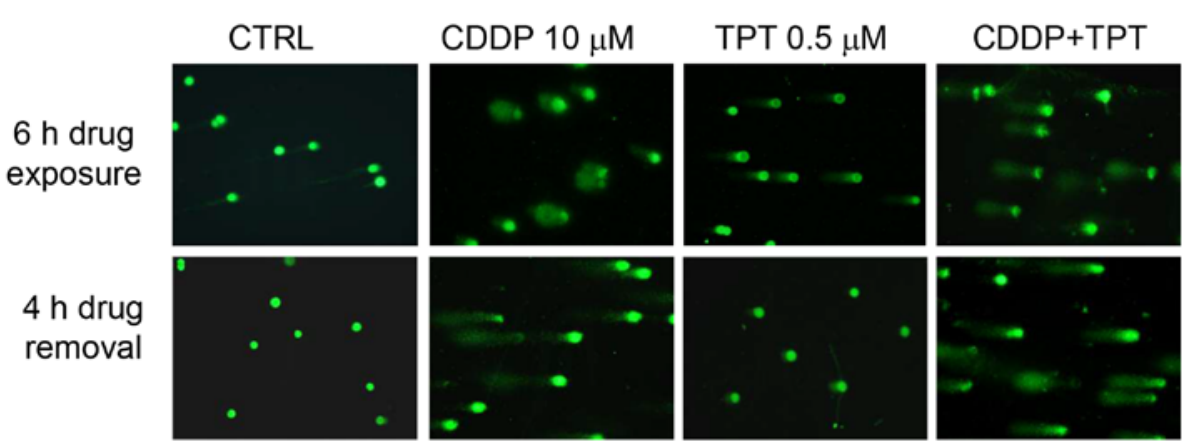

C

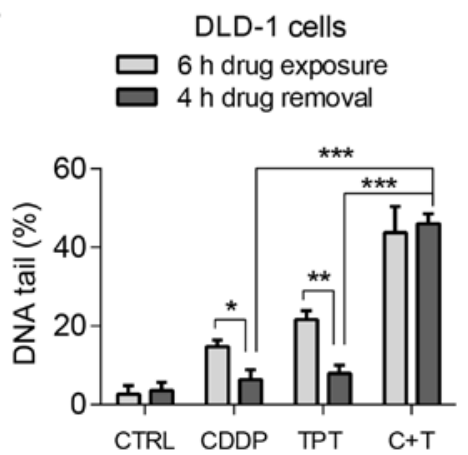

D

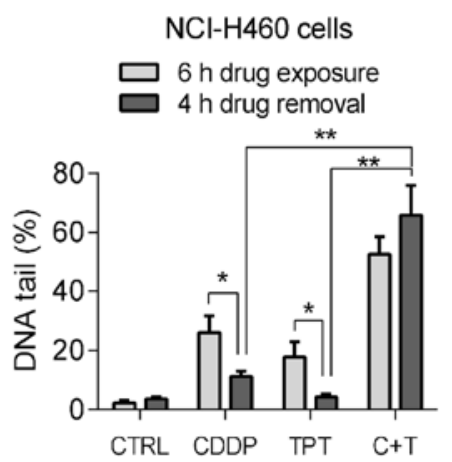

Figure 4. Effect of topotecan on DNA damage induced by cisplatin (CDDP). (A) Comet assay of DNA damage in DLD-1 cells treated with CDDP (10 $\mu$ M), topotecan $(1 \mu \mathrm{M})$, or their combination. Cells were treated with the indicated concentrations of the drugs for $6 \mathrm{~h}$, and the samples were then either subjected to comet assay or incubated in drug-free medium for another $4 \mathrm{~h}$ to allow potential DNA repair. The bright green dots represent the cellular nuclei area; the 'tail' length and intensity on the left side of each nucleus represent the degree of DNA fragmentations eluted out from the cell during electrophoresis. (B) Comet assay of DNA damage in NCI-H460 cells treated with the indicated concentrations of CDDP, topotecan $(0.5 \mu \mathrm{M})$, or their combination. (C) Quantitative data of DNA damage in DLD-1 cells treated with CDDP $(10 \mu \mathrm{M})$, topotecan $(1 \mu \mathrm{M})$, or their combination. The comparison between the 6-h drug exposure group and the 4-h drug removal group within each independent treatment (CDDP, TPT or C $+\mathrm{T}$ ) was analyzed by a Student's t-test. The comparison of (C + T) vs. the CDDP group or $(\mathrm{C}+\mathrm{T})$ vs. the TPT group was analyzed by the Student's t-test with Bonferroni correction. (D) Quantitative data of DNA damage in NCI-H460 cells treated with CDDP $(10 \mu \mathrm{M})$, topotecan $(0.5 \mu \mathrm{M})$, or their combination. The comparison between the 6-h drug exposure group and the 4-h drug removal group within each independent treatment (CDDP, TPT, or C $+\mathrm{T}$ ) was analyzed by the Student's t-test. The comparison of $(\mathrm{C}+\mathrm{T})$ vs. the CDDP group or $(\mathrm{C}+\mathrm{T})$ vs. the TPT group was analyzed by the Student's t-test with Bonferroni correction. Data are shown as the means $\pm \mathrm{SD}, \mathrm{n}=4$ experiments. ${ }^{*} \mathrm{P}<0.05 ;{ }^{* * *} \mathrm{P}<0.01 ;{ }^{* * * *} \mathrm{P}<0.001$ following Bonferroni correction.

Topotecan impairs the ability of cells to repair CDDP-induced DNA damage. The cytotoxicity of CDDP is mainly induced by DNA damage from the formation of drug-DNA adducts, leading to interstrand and intrastrand crosslinks, and doublestrand breaks (DSBs) (5). Considering the important role of topoisomerase I, a therapeutic target of topotecan, in chromatin remodeling and DNA repair (25-27), we hypothesized that the CDDP-induced DNA damage may be enhanced by topotecan due to its inhibition of topoisomerase I. Using the alkaline comet assay, we observed that DNA strand breaks induced by CDDP were significantly increased by topotecan, as evidenced by the appearance of increased comet tails after single-cell gel electrophoresis (Fig. 4A and B). In the cells treated with CDDP alone, the drug-induced DNA damage was largely repaired at $4 \mathrm{~h}$ after the removal of CDDP, as evidenced by the disappearance or reduction in the number of DNA tails. The addition of topotecan rendered the cells unable to repair the DNA damage, resulting in the persistence of strand breaks 

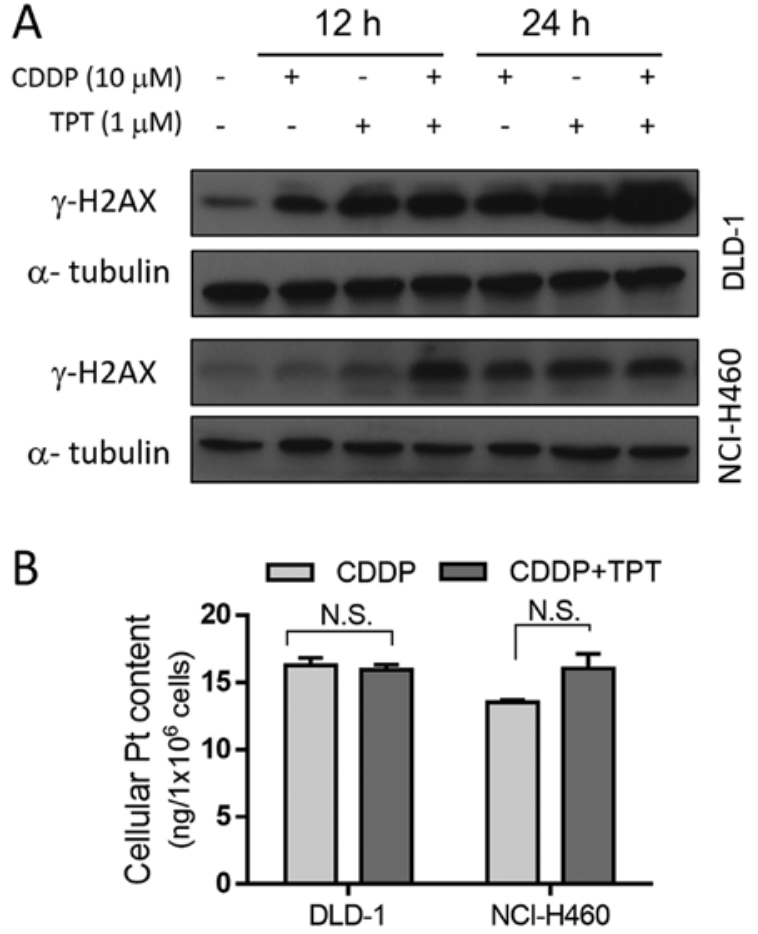

Figure 5. Effect of topotecan on cisplatin (CDDP)-induced $\gamma$-H2AX expression and cellular Pt content. (A) Western blot analysis of $\gamma$-H2AX expression induced by CDDP, topotecan and their combination as indicated. (B) Total intracellular Pt content in DLD-1 and NCI-H460 after $24 \mathrm{~h}$ incubation with CDDP with or without topotecan. Data are shown as the means \pm SD $(n=3$ experiments); N.S., not significant.

(comet tails) at $4 \mathrm{~h}$ after drug removal. The quantitative data for the DNA strand breaks are presented in Fig. 4C and D.

Topotecan enhances the formation of CDDP-induced DNA $D S B s$ without affecting cellular Pt content. The replication DSBs produce several well-characterized molecular responses, including the phosphorylation of the H2AX histone variant, which occurs within minutes after the formation of DSBs (28). The phosphorylated form of $\mathrm{H} 2 \mathrm{AX}$, termed $\gamma-\mathrm{H} 2 \mathrm{AX}$, can be detected by immunofluorescence or immunostaining as it accumulates and forms nuclear foci at DSBs (29). Thus, we used western blot analysis of $\gamma-\mathrm{H} 2 \mathrm{AX}$ to detect the DSBs induced by CDDP in the presence and absence of topotecan. The $\gamma-\mathrm{H} 2 \mathrm{AX}$ level increased after each of the drug treatments alone, and was further enhanced when the two drugs were combined (Fig. 5A). The results revealed that DNA damage at $24 \mathrm{~h}$ was significantly more severe when the DLD-1 cells were treated with both CDDP and topotecan compared with when they were treated with either drug alone. A similar combination result was observed in the NCI-H460 cells following treatment with the drugs for $12 \mathrm{~h}$.

As nucleotide excision repair (NER) is the primary mechanism for the removal of CDDP from the cell nucleus (30), and Pt efflux is a critical mechanism of resistance to CDDP (31), the impact of topotecan on cellular Pt content was measured to examine the possibility that topotecan may enhance the anticancer activity of CDDP by increasing cellular Pt content. Quantitative analysis of Pt revealed that, following a 24-h incubation of the DLD-1 cells with $10 \mu \mathrm{M}$ CDDP in the presence or absence of topotecan, there was no difference in intracellular
Pt content between the two groups (Fig. 5B). Similar results were also observed in the NCI-H460 cells (Fig. 5B). These results indicate that the increase in DNA DSBs in cells treated with both CDDP and topotecan was not due to an increase in the cellular accumulation of $\mathrm{Pt}$, but was likely due to the inhibition of DNA repair.

\section{Discussion}

CDDP-based chemotherapy is one of the conventional regimens used in the clinical treatment of various cancers of different tissue types. However, the development of drug resistance and toxic side-effects pose major challenges to the clinical use of CDDP (1-3,6,7). Even with newer-generation Pt drugs, such as oxaliplatin and carboplatin, cross-resistance and toxic effects still develop $(32,33)$. Thus, an effective regimen combining CDDP with another clinical drug to overcome resistance to treatment and reduce the incidence and severity of toxic sideeffects is urgently required. Various high-throughput screening technologies for the discovery of potential drug combination regimens have been reported (34), although there remain challenges in establishing effective combination screening.

In the present study, we were able to identify clinical drugs that enhance the anticancer activity of CDDP by using a cell-based assay to screen a collection of FDA-approved drugs. Among the library of 1,280 FDA-approved compounds screened, 10 drugs exhibited potential ability to enhance the cytotoxicity of CDDP. One of the compounds identified, topotecan, has previously been demonstrated to improve the therapeutic efficacy when combined with CDDP in the clinical treatment of cancers (35). Thus, it appears that this cell-based screening strategy is robust and may yield clinically relevant hits. Therefore, it is possible to use this method to identify FDA-approved drugs that may improve the therapeutic efficacy of other chemotherapeutic agents.

PAT was the top hit from our screening assay. This compound, also known as tartar emetic, was previously used as an anti-parasitic agent for the treatment of leishmaniosis and schistosomiasis $(36,37)$. PAT, similar to other metal-containing compounds, such as arsenic trioxide and CDDP, possesses anticancer properties and has been proposed as a potential novel therapy for acute promyelocytic leukemia and other malignancies (38). It has been reported that PAT may induce the caspase- and reactive oxygen species (ROS)-dependent apoptosis of human myeloid leukemia HL-60 cells and lymphoid tumor cells $(39,40)$. Of note, a recent study reported that PAT exerted anti-angiogenic effects on lung cancer (41). The present study revealed the significant ability of PAT to enhance the anticancer activity of CDDP in a synergistic manner, suggesting that this drug may potentially be used in combination with CDDP to improve the therapeutic efficacy. It should be noted, however, that PAT is a strong emetic agent that may induce vomiting $(42,43)$, and such an effect may limit its potential use as an anticancer agent in combination with CDDP, which is also known to cause nausea and vomiting. The mechanisms through which PAT enhanced the anticancer activity of CDDP are currently unclear. One possibility is that PAT-induced ROS generation $(39,40)$ may increase the DNA damage induced by CDDP. Evidently, further investigation is required to gain further insight into the underlying 
mechanisms, which may serve as a basis for developing more effective CDDP sensitizers.

Topotecan [10-hydroxy-9-dimethylaminomethyl-(S)camptothecin] is an inhibitor of topoisomerase I, and has been used in the clinical treatment of ovarian, cervical and lung cancer. This compound exerts its cytotoxic effects by inhibiting the enzymatic complex DNA-topoisomerase I in the nucleus, thus blocking the normal DNA replication process $(44,45)$. Previous studies have revealed that topotecan may potentiate the cytotoxic activity of CDDP, etoposide and paclitaxel in cervical cancer cell lines $(46,47)$. The combination of CDDP and topotecan as a clinical regimen is based on the hypothesis that the concomitant administration of these two agents is advantageous compared with either agent alone (48). This drug combination was approved by the FDA in 2006 for the treatment of patients with advanced-stage cervical carcinoma who were unsuitable for surgery or radiation therapy (49). Our unbiased cell-based screening identified topotecan as one of the top hits that synergistically enhanced the anticancer activity of CDDP in both lung and colon cancer cells. Further mechanistic analyses demonstrated that topotecan was able to enhance the number of DNA DSBs induced by CDDP, and inhibited the repair of DNA stand breaks after drug removal without affecting the intracellular Pt content.

In conclusion, the findings of this study suggest that the cell-based high-throughput screening of the FDA-approved drugs in combination with a major chemotherapeutic agent represents an effective strategy for the identification of synergistic drug combination regimens with feasibility to be translated into the clinical treatment of cancer patients. Further mechanistic studies of the identified drug combinations may provide valuable new information to serve as a basis for developing more effective chemosensitizers and improve the clinical outcome for cancer patients.

\section{Acknowledgements}

The authors would like to acknowledge Dr Huaiqiang Ju and Ms. Alice Qiu for providing technical assistance, as well as Ms. Ting Li and Dr Yongqiao He for their advice on statistical analysis.

\section{Funding}

This study was supported in part by grants from the National Natural Science Foundation of China (no. 81430060), Guangzhou Technology Innovation Research Program (nos. 201508020250; 201504010038; and LCY201317).

\section{Availability of data and materials}

The analyzed datasets generated during the study are available from the corresponding author on reasonable request.

\section{Authors' contributions}

YS and WJ conducted most of the experiments in this study with some assistance from JL and PJC. WL and MS carried out apoptosis analyses. KL, PC and AC conducted cellular Pt content analysis. AC, JL and PJC provided inputs on study design and data interpretation, and critically reviewed the manuscript. PH and YH designed and conceived the study. YS, YH and PH analyzed all the data, and wrote the manuscript. All authors have read and approved the final manuscript.

\section{Ethics approval and consent to participate}

Not applicable.

\section{Consent for publication}

Not applicable.

\section{Competing interests}

The authors declare that they have no competing interests.

\section{References}

1. Rosenberg B, VanCamp L, Trosko JE and Mansour VH: Platinum compounds: A new class of potent antitumour agents. Nature 222: 385-386, 1969.

2. Jamieson ER and Lippard SJ: Structure, recognition, and processing of cisplatin-DNA adducts. Chem Rev 99: 2467-2498, 1999.

3. Giacchetti S, Perpoint B, Zidani R, Le Bail N, Faggiuolo R, Focan C, Chollet P, Llory JF, Letourneau Y, Coudert B, et al: Phase III multicenter randomized trial of oxaliplatin added to chronomodulated fluorouracil-leucovorin as first-line treatment of metastatic colorectal cancer. J Clin Oncol 18: 136-147, 2000

4. Vasey PA, Paul J, Birt A, Junor EJ, Reed NS, Symonds RP, Atkinson R, Graham J, Crawford SM, Coleman R, et al; Scottish Gynaecological Cancer Trials Group: Docetaxel and cisplatin in combination as first-line chemotherapy for advanced epithelial ovarian cancer. J Clin Oncol 17: 2069-2080, 1999.

5. Wang D and Lippard SJ: Cellular processing of platinum anticancer drugs. Nat Rev Drug Discov 4: 307-320, 2005.

6. Kelland L: The resurgence of platinum-based cancer chemotherapy. Nat Rev Cancer 7: 573-584, 2007.

7. Santos NA, Catão CS, Martins NM, Curti C, Bianchi ML and Santos AC: Cisplatin-induced nephrotoxicity is associated with oxidative stress, redox state unbalance, impairment of energetic metabolism and apoptosis in rat kidney mitochondria. Arch Toxicol 81: 495-504, 2007.

8. Dasari S and Tchounwou PB: Cisplatin in cancer therapy: Molecular mechanisms of action. Eur J Pharmacol 740: 364-378, 2014.

9. Macciò A and Madeddu C: Cisplatin : An old drug with a newfound efficacy - from mechanisms of action to cytotoxicity. Expert Opin Pharmacother 14: 1839-1857, 2013.

10. Holohan C, Van Schaeybroeck S, Longley DB and Johnston PG: Cancer drug resistance: An evolving paradigm. Nat Rev Cancer 13: 714-726, 2013

11. Kelland LR, Sharp SY, O'Neill CF, Raynaud FI, Beale PJ and Judson IR: Mini-review: Discovery and development of platinum complexes designed to circumvent cisplatin resistance. J Inorg Biochem 77: 111-115, 1999.

12. Wong E and Giandomenico CM: Current status of platinumbased antitumor drugs. Chem Rev 99: 2451-2466, 1999.

13. Martín M: Platinum compounds in the treatment of advanced breast cancer. Clin Breast Cancer 2: 190-209, 2001.

14. Cosaert $\mathrm{J}$ and Quoix E: Platinum drugs in the treatment of nonsmall-cell lung cancer. Br J Cancer 87: 825-833, 2002.

15. Al-Lazikani B, Banerji U and Workman P: Combinatorial drug therapy for cancer in the post-genomic era. Nat Biotechnol 30: 679-692, 2012.

16. Chong CR and Sullivan DJ Jr: New uses for old drugs. Nature 448 645-646, 2007.

17. Weir SJ, DeGennaro LJ and Austin CP: Repurposing approved and abandoned drugs for the treatment and prevention of cancer through public-private partnership. Cancer Res 72: 1055-1058, 2012

18. Pessetto ZY, Weir SJ, Sethi G, Broward MA and Godwin AK Drug repurposing for gastrointestinal stromal tumor. Mol Cancer Ther 12: 1299-1309, 2013 
19. Chou TC: Drug combination studies and their synergy quantification using the Chou-Talalay method. Cancer Res 70: 440-446, 2010.

20. Mathews Griner LA, Guha R, Shinn P, Young RM, Keller JM, Liu D, Goldlust IS, Yasgar A, McKnight C, Boxer MB, et al: High-throughput combinatorial screening identifies drugs that cooperate with ibrutinib to kill activated B-cell-like diffuse large B-cell lymphoma cells. Proc Natl Acad Sci USA 111: 2349-2354, 2014.

21. Yuan S, Wang F, Chen G, Zhang H, Feng L, Wang L, Colman H, Keating MJ, Li X, Xu RH, et al: Effective elimination of cancer stem cells by a novel drug combination strategy. Stem Cells 31: 23-34, 2013.

22. Di Pasqua AJ, Hong C, Wu MY, McCracken E, Wang X, Mi L and Chung FL: Sensitization of non-small cell lung cancer cells to cisplatin by naturally occurring isothiocyanates. Chem Res Toxicol 23: 1307-1309, 2010.

23. Spigel DR and Greco FA: Chemotherapy in metastatic and locally advanced non-small cell lung cancer. Semin Surg Oncol 21: 98-110, 2003

24. Hata F, Sasaki K, Hirata K, Yamamitsu S and Shirasaka T: Efficacy of a continuous venous infusion of fluorouracil and daily divided dose cisplatin as adjuvant therapy in resectable colorecta cancer: A prospective randomized trial. Surg Today 38: 623-632, 2008.

25. Pommier Y: Topoisomerase I inhibitors: Camptothecins and beyond. Nat Rev Cancer 6: 789-802, 2006.

26. Wang JC: Cellular roles of DNA topoisomerases: A molecular perspective. Nat Rev Mol Cell Biol 3: 430-440, 2002.

27. Staker BL, Hjerrild K, Feese MD, Behnke CA, Burgin AB Jr and Stewart L: The mechanism of topoisomerase I poisoning by a camptothecin analog. Proc Natl Acad Sci USA 99: 15387-15392, 2002.

28. Redon C, Pilch D, Rogakou E, Sedelnikova O, Newrock K and Bonner W: Histone H2A variants H2AX and H2AZ. Curr Opin Genet Dev 12: 162-169, 2002.

29. Furuta T, Takemura H, Liao ZY, Aune GJ, Redon C, Sedelnikova OA, Pilch DR, Rogakou EP, Celeste A, Chen HT, et al: Phosphorylation of histone $\mathrm{H} 2 \mathrm{AX}$ and activation of Mre11, Rad50, and Nbs1 in response to replication-dependent DNA double-strand breaks induced by mammalian DNA topoisomerase I cleavage complexes. J Biol Chem 278: 20303-20312, 2003.

30. Costa RM, Chiganças V, Galhardo RS, Carvalho H and Menck CF: The eukaryotic nucleotide excision repair pathway. Biochimie 85: 1083-1099, 2003.

31. Komuro Y, Udagawa Y, Susumu N, Aoki D, Kubota T and Nozawa S: Paclitaxel and SN-38 overcome cisplatin resistance of ovarian cancer cell lines by down-regulating the influx and efflux system of cisplatin. Jpn J Cancer Res 92: 1242-1250, 2001.

32. Stordal B, Pavlakis N and Davey R: Oxaliplatin for the treatment of cisplatin-resistant cancer: A systematic review. Cancer Treat Rev 33: 347-357, 2007.

33. Terakawa T, Miyake H, Yokoyama N, Miyazaki A, Tanaka H, Inoue $\mathrm{T}$ and Fujisawa M: Clinical outcome of paclitaxel and carboplatin as second-line chemotherapy for advanced urothelial carcinoma resistant to first-line therapy with gemcitabine and cisplatin. Urol Int 92: 180-185, 2014.

34. Small BG, McColl BW, Allmendinger R, Pahle J, LópezCastejón G, Rothwell NJ, Knowles J, Mendes P, Brough D and Kell DB: Efficient discovery of anti-inflammatory smallmolecule combinations using evolutionary computing. Nat Chem Biol 7: 902-908, 2011.
35. Pignata S, Cannella L, Leopardo D, Pisano C, Bruni GS and Facchini G: Chemotherapy in epithelial ovarian cancer. Cancer Lett 303: 73-83, 2011.

36. Murray HW, Pépin J, Nutman TB, Hoffman SL and Mahmoud AA: Tropical medicine. BMJ 320: 490-494, 2000.

37. Schulert AR, Rassoul AA, Mansour M, Girgis N, McConnell E and Farid Z: Biological disposition of antibilharzial antimony drugs. II. Antimony fate and uptake by Schistosoma haematobium eggs in man. Exp Parasitol 18: 397-402, 1966.

38. Duffin J and Campling BG: Therapy and disease concepts: The history (and future?) of antimony in cancer. J Hist Med Allied Sci 57: 61-78, 2002.

39. Lecureur V, Lagadic-Gossmann D and Fardel O: Potassium antimonyl tartrate induces reactive oxygen species-related apoptosis in human myeloid leukemic HL60 cells. Int J Oncol 20: 1071-1076, 2002

40. Lecureur V, Le Thiec A, Le Meur A, Amiot L, Drenou B, Bernard M, Lamy T, Fauchet R and Fardel O: Potassium antimonyl tartrate induces caspase- and reactive oxygen species-dependent apoptosis in lymphoid tumoral cells. Br J Haematol 119: 608-615, 2002.

41. Wang B, Yu W, Guo J, Jiang X, Lu W, Liu M and Pang X: The antiparasitic drug, potassium antimony tartrate, inhibits tumor angiogenesis and tumor growth in nonsmall-cell lung cancer. J Pharmacol Exp Ther 352: 129-138, 2015.

42. Weiss $\mathrm{S}$ and Hatcher RA: The mechanism of the vomiting induced by antimony and Potassium tartrate (Tartar emetic). J Exp Med 37: 97-111, 1923.

43. Haldar AK, Sen P and Roy S: Use of antimony in the treatment of leishmaniasis: Current status and future directions. Mol Biol Int 2011: 571242, 2011.

44. Robati M, Holtz D and Dunton CJ: A review of topotecan in combination chemotherapy for advanced cervical cancer. Ther Clin Risk Manag 4: 213-218, 2008.

45. Riemsma R, Simons JP, Bashir Z, Gooch CL and Kleijnen J: Systematic review of topotecan (Hycamtin) in relapsed small cell lung cancer. BMC Cancer 10: 436, 2010.

46. Boabang P, Kurbacher CM, Kohlhagen H, Waida A and Amo-Takyi BK: Anti-neoplastic activity of topotecan versus cisplatin, etoposide and paclitaxel in four squamous cell cancer cell lines of the female genital tract using an ATP-tumor chemosensitivity assay. Anticancer Drugs 11: 843-848, 2000.

47. Chou TC, Motzer RJ, Tong Y and Bosl GJ: Computerized quantitation of synergism and antagonism of taxol, topotecan, and cisplatin against human teratocarcinoma cell growth: A rational approach to clinical protocol design. J Natl Cancer Inst 86: 1517-1524, 1994.

48. Fiorica JV: The role of topotecan in the treatment of advanced cervical cancer. Gynecol Oncol 90: S16-S21, 2003.

49. Brave M, Dagher R, Farrell A, Abraham S, Ramchandani R, Gobburu J, Booth B, Jiang X, Sridhara R, Justice R, et al: Topotecan in combination with cisplatin for the treatment of stage IVB, recurrent, or persistent cervical cancer. Oncology 20: 1401-1404, 1410-1411, 1415-1406, 2006. 